\title{
Gas plasma sterilization of microorganisms and mechanisms of action (Review)
}

\author{
HIDEHARU SHINTANI $^{1}$, AKIKAZU SAKUDO ${ }^{2}$, PETER BURKE $^{3}$ and GERALD McDONNELL ${ }^{4}$ \\ ${ }^{1}$ Faculty of Science and Engineering, Chuo University, Tokyo 112-8551; ${ }^{2}$ Laboratory of Biometabolic Chemistry, \\ School of Health Sciences, Faculty of Medicine, University of the Ryukyus, Okinawa 903-0215, Japan; \\ ${ }^{3}$ STERIS Corporation, Mentor, OH 44060, USA; ${ }^{4}$ STERIS Ltd., Hampshire RG22 4AX, UK
}

Received June 8, 2010; Accepted July 6, 2010

DOI: $10.3892 /$ etm.2010.136

\begin{abstract}
The use of true gas plasmas for the inactivation of microorganisms is an area of dynamic research. Many types of gases are used as a source of plasma, and different plasma production methods have been applied. The antimicrobial mechanisms of oxygen-based gas plasmas may be due to an etching effect on microbial structures, particularly bacterial endospores resulting in shrinkage. By contrast, the definite mechanisms of actions of other gas plasma sources, such as $\mathrm{N}_{2}, \mathrm{He}, \mathrm{Ne}, \mathrm{Ar}$ and Xe gases, have not been clearly defined and indeed may be distinct. The speculated mechanisms of these gas plasmas involve the direct attack of metastable (excited molecular), UV and/or VUV to microbial structures, specifically the inner membrane and DNA in the core of bacterial endospores. According to this speculation, sterilized spore figures would remain unchanged. However, these mechanisms remain to be clarified. Future perspectives on the use of gas plasma for sterilization are of interest, as it is possible that appropriate sterility assurance levels can be obtained in parallel with material and functional compatibility. Traditional sterilization methods are often limited in these requirements. Therefore, gas plasma sterilization may prove to be an appropriate alternative sterilization procedure.
\end{abstract}

\section{Contents}

1. Introduction

2. Active species for sterilization by gas plasma

3. Current uses of plasma for decontamination

4. Speculated mechanism of the action of gas plasma

5. Sporicidal test and the importance of spore clumping

6. Perspectives for the future

Correspondence to: Dr Hideharu Shintani, Faculty of Science and Engineering, Chuo University, 1-13-27 Kasuga, Bunkyo, Tokyo 112-8551, Japan

E-mail: shintani@mail.hinocatv.ne.jp

Key words: gas plasma sterilization, oxygen gas, rare gases, etching, vacuum UV

\section{Introduction}

Plasma is defined as an ionized (or energized) gas with an equal number of positively and negatively charged particles. Plasma is often regarded as the 'fourth state of matter' (the other three being solids, liquids and gases) because, while plasma is neither a gas nor a liquid, its properties are similar to those of both gases and liquids. Plasma can be categorized as either high-temperature- or low-temperature-based. A good example of a naturally occurring high-temperature plasma is lightning. This type of plasma can be artificially generated using a high-voltage high-temperature arc, which is the basis for the corona discharge process and for plasma torches used to vaporize and redeposit metals. Low-temperature plasmas, used in surface modification, cleaning, decontamination and sterilization applications, are ionized gases generated under deep vacuum (low-pressure) conditions. These types of plasmas operate within a vacuum chamber in which atmospheric gases have been introduced into the chamber typically evacuated below 0.1 Torr. Such low pressures allow for a relatively long free path of accelerated electrons and ions within the chamber. Since these ions and neutral particles are at or near ambient temperatures and the long free path of electrons at a high temperature or at electron volt levels have relatively few collisions with molecules at this pressure, the overall exposure conditions remain at a low temperature.

For some applications, deep vacuum pressure sterilization cannot be used, and atmospheric plasmas have been proposed. The lifetime of the reactive plasma species under atmospheric conditions is much shorter than that of low-pressure plasma. As a result, most of the plasma active species, with the exception of the metastables (long-life electronically excited atoms), lose their reactivity dramatically in a remote position. According to one report, atmospheric or one third atmospheric pulsed plasma equipment may be more desirable (1).

It is important to note that 'true' plasma decontamination processes have not been widely used to date. A variety of hydrogen peroxide gas-based processes that use plasma as part of the sterilization process are instead commonly regarded as 'plasma sterilization'. However, it is now known that the mechanisms of antimicrobial action in these processes are primarily due to hydrogen peroxide gas (or condensed gas) and not plasma, although the plasma generation phase of these 
processes is known to assist in the breakdown of residual peroxide in the chamber load (2).

There are various reports regarding the potential use of plasmas for sterilization applications, in particular the demonstration of broad spectrum antimicrobial (particularly sporidical) activity and the kinetics of this activity over time. This is an important requirement for the demonstration of any sterilization process as described in international standards (3). Several types of source gases and production procedures of gas plasma have been reported and discussed $(4,5)$. As a focus in this review article, we consider a number of variables that may have been overlooked and misunderstood in previous studies. One is the tailing phenomenon observed in microbial inactivation studies. This is an important variable in the design of a typical overkill sterilization process, and may be due to a number of factors, including artifacts in the test method, such as spore clumping in a biological indicator (BI). Spore clumping may have the same effects as organic matter in interfering with the sterilization efficiency of gas plasma under such investigations. The tailing phenomenon commonly reported for plasma sterilization investigations has been further investigated and clarified using clumping-free BIs. A further consideration is the use of so-called 'inert gas plasma sterilization' and differences in its mechanisms of action. The use of inert gases is quite desirable, since they are believed to attain the required overkill in the sterilization process [such as a sterility assurance level (SAL) of at least $10^{-6}$ ] in parallel with material and functional compatibility. This is an important balance to achieve for sterilization validation in medical and manufacturing applications. Inert gas plasma applications are the focus of further investigation, patenting and discussion worldwide.

\section{Active species for sterilization by gas plasma}

Several types of active species are considered to contribute to the antimicrobial effects of gas plasma. They include various types of ions, electrons, radicals, UV light, vacuum UV (VUV), electric fields and metastables (Figs. 1 and 2) (http:// www.astp.com/plasma-equipment/applications). It is important to note that these active species may differ depending on the types of gas used for plasma formation and the methods used to generate the plasma. These are further discussed below, based on the various types of gas commonly used.

Oxygen. The most popular gas investigated to date for plasma sterilization is oxygen $\left(\mathrm{O}_{2}\right) . \mathrm{O}_{2}$ produces $\mathrm{O}, \mathrm{OH}, \mathrm{OOH}$ and a mixture of other radicals that contribute to the sterilization effects (6). Among these, the $\mathrm{OH}$ radical is often considered the most efficient for microbial inactivation, despite its extremely short lifetime (6) (Fig. 3).

The mechanism of various $\mathrm{O}_{2}$ radicals is believed to be due to etching effects (Fig. 2). Based on direct visualization of exposed bacterial endospores, the greater the amount of $\mathrm{O}_{2}$ plasma exposure, the more shrinkage of spores is observed (Fig. 4) (5). There is a significant relationship between the degree of shrinkage and spore death (Fig. 5) (7); the coefficient relationship of the regression line as shown in Fig. 5 is 0.949. By contrast, $\mathrm{N}_{2}$ plasma does not present any shrinkage $(7,8)$. The difference in the mechanisms of $\mathrm{O}_{2}$ and $\mathrm{N}_{2}$ gases has

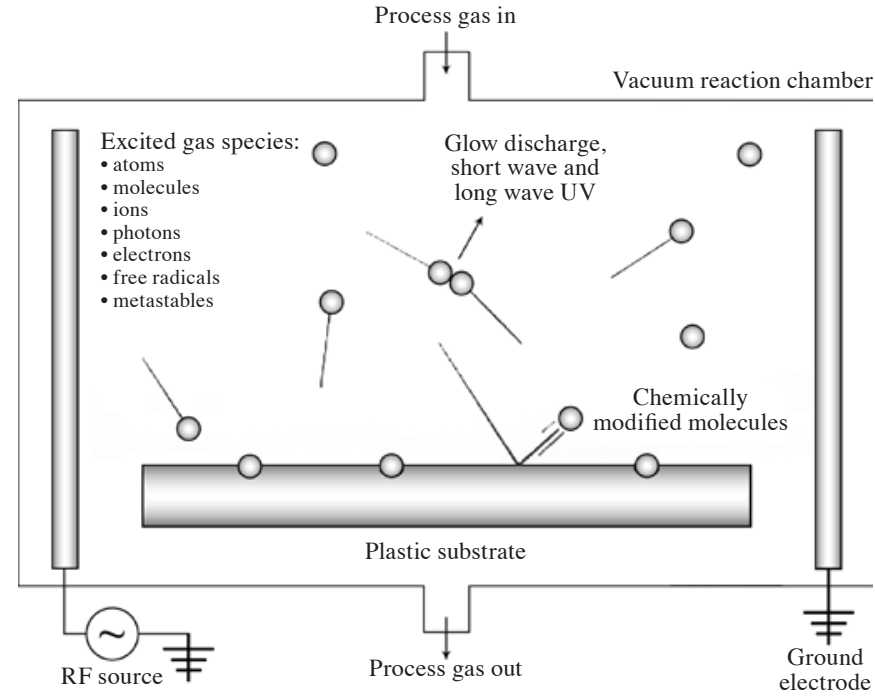

Figure 1. Model case of produced chemical species and UV exposed by gas plasma.

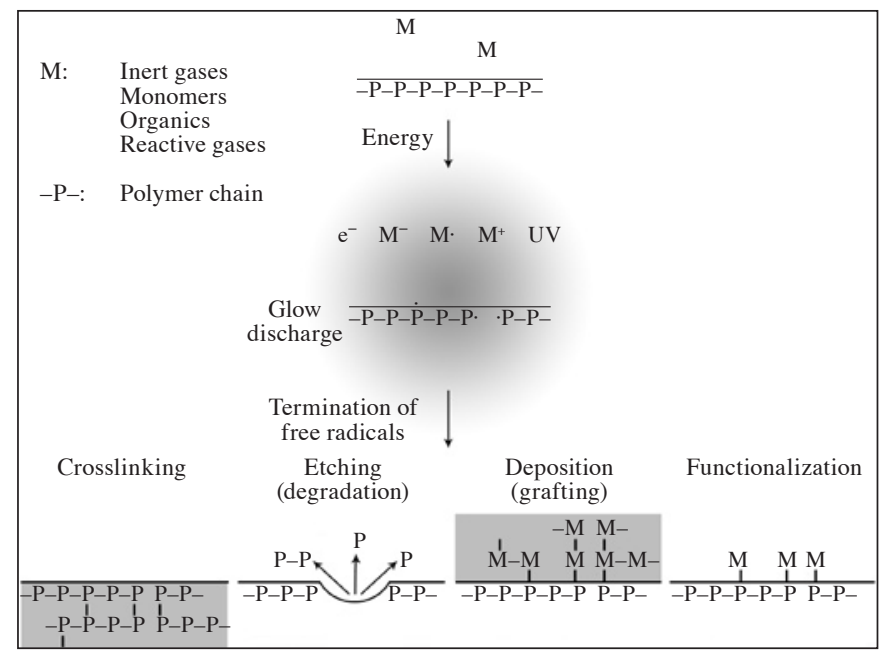

Figure 2. Speculated model of chemical reactions exposed by gas plasma.

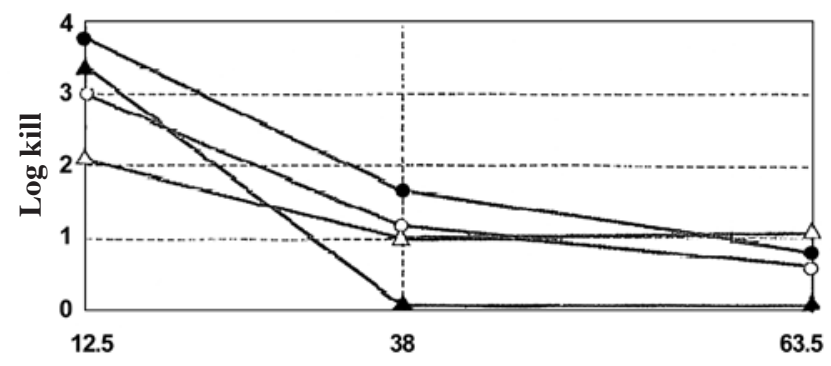

Figure 3. Theoretical comparison of the antimicrobial activity of $\mathrm{O}(\bullet), \mathrm{OH}$ $(\mathbf{\wedge}), \mathrm{OOH}(0)$ and $\mathrm{H}(\triangle)$ radicals $(6)$.

yet to be clarified, and should be seriously considered from an antimicrobial and compatibility point of view.

Nitrogen. As demonstrated by Rossi et al $(7,8)$, upon $\mathrm{N}_{2}$ gas plasma generation the active species had no or insufficient ability to shrink bacterial endospores, suggesting a different 


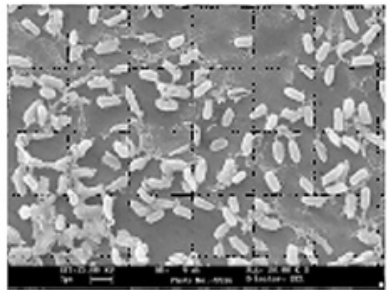

$\mathrm{O}_{2}$

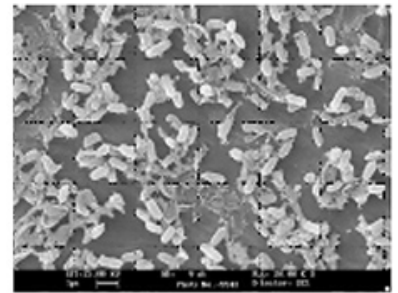

$\mathrm{O}_{2}: \mathrm{N}_{2} 80: 20$

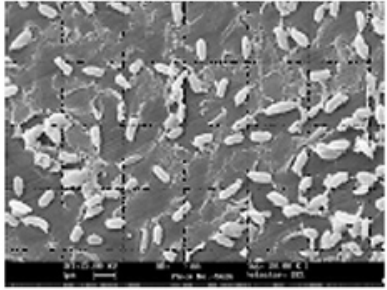

$\mathrm{O}_{2}: \mathrm{N}_{2}$ 90:10

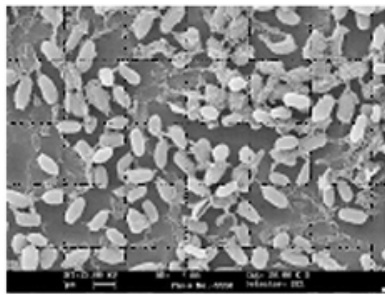

$\mathrm{O}_{2}: \mathrm{N}_{2} \mathbf{5 0 : 5 0}$
Figure 4. Scanning electron microscopic observations of bacterial spores upon exposure to several ratios of nitrogen/oxygen mixtures (5).

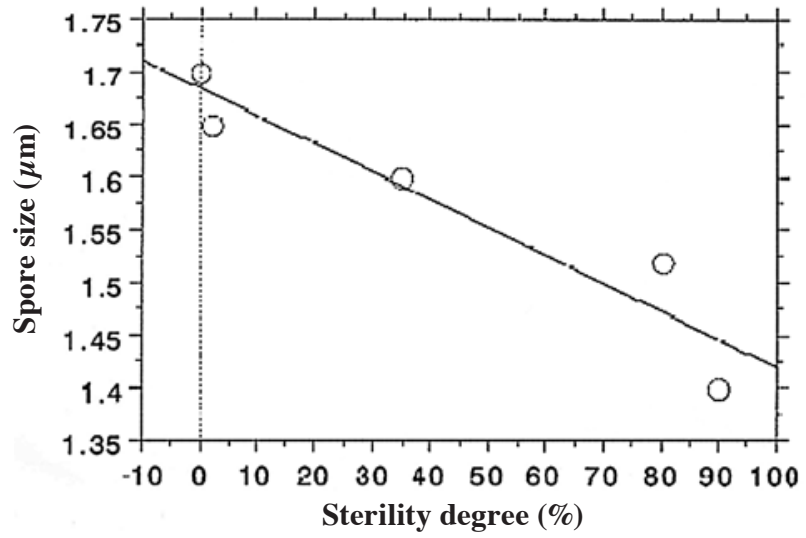

Figure 5. Percent sterility degree of a spore population (\%) vs. spore size $(\mu \mathrm{m})$ upon treatment with gas plasma. Original data is from Rossi et al (7). A coefficient relationship of 0.949 between the scale of shrinkage and the rate of spore death was noted.

mechanism of action than that at play with oxygen. There are currently no specific theories as to why this is the case, but it may be due to the difficulty of ionizing $\mathrm{N}_{2}$. Chemically, $\mathrm{N}_{2}$ is a molecule with a triple bond, so the dissociation energy is high - approximately $9.91 \mathrm{eV}(1)$ - suggesting it is resistant to ionization (Table I). From this, it can be speculated that $\mathrm{N}^{+}$or $\mathrm{N}^{*}$ ions are hard to practically attain in comparison to oxygen. However, it is not known which active species are present to cause the sterilization effects observed with $\mathrm{N}_{2}$ gas plasmas. UV-C (254 nm) (Fig. 6) (http://www.phys.ksu.edu/gene/f_5. html) may be present, but in amounts that are considered to be low (1); VUV (<200 nm) may also be present, but this remains to be definitively confirmed.

If we consider the effects observed with pulsed plasma processes (1), the direct or contributory effects of the pulsed voltage may lead to direct structural changes in spores, where many cracks are observed on microscopic analysis (1) (Fig. 7). Overall, whether such physical factors significantly contribute to these effects is difficult to assess, and further research is

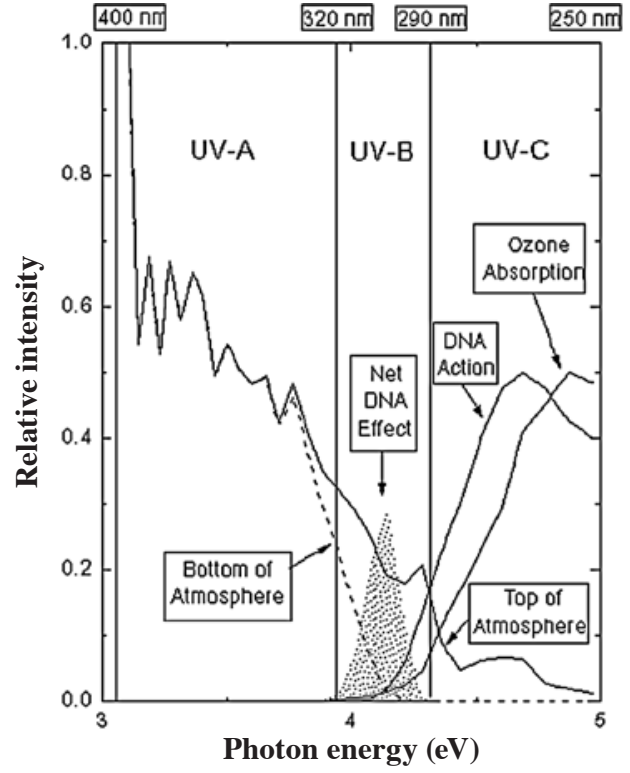

Figure 6. The effects of UV-A, UV-B and UV-C showing optimal activity against DNA in the UV-C wavelength band (from http://www.phys.ksu.edu/ gene/f5.html).

Table I. Dissociation energy of several sorts of gases.

Gas

Dissociation energy (eV)

\begin{tabular}{ll}
\hline $\mathrm{N}_{2}$ & 9.91 \\
$\mathrm{O}_{2}$ & 5.21 \\
$\mathrm{H}_{2} \mathrm{O}$ & 5.11 \\
$\mathrm{NO}$ & 6.50 \\
$\mathrm{SO}_{2}$ & 5.60 \\
$\mathrm{~N}_{2} \mathrm{O}$ & 4.93 \\
$\mathrm{CO}_{2}$ & 5.52 \\
$\mathrm{O}_{3}$ & 1.05 \\
$\mathrm{H}_{2} \mathrm{O}_{2}$ & 2.21 \\
\hline
\end{tabular}

Original data is from Shintani et al (1).

needed to elucidate the true antimicrobial effects and active species involved in $\mathrm{N}_{2}$ gas plasmas.

Penetration depth of active species in $\mathrm{O}_{2}$ and $\mathrm{N}_{2}$ gas plasmas. The penetration depth of active species (ions and radicals; Figs. 1 and 2) in the case of $\mathrm{N}_{2}$ gas appears to be approximately $10-40 \mathrm{~nm}$ from the surface of spores, as observed by atomic force microscopy (Fig. 8) (1).

Fig. 8 displays the effect of $\mathrm{N}_{2}$ gas plasma exposure in contrast to $\mathrm{O}_{2}$ gas plasma exposure, which appears to have a somewhat deeper penetrating ability and more extensive effects on bacterial spores $(7,8)$. Since the scale of a typical spore structure is $1,000 \times 3,000 \mathrm{~nm}$, the penetration depth of $\mathrm{O}_{2}$ gas plasma was predicted to be $<1,000 \mathrm{~nm}$. This suggests that it penetrates ten times deeper than $\mathrm{N}_{2}$ gas plasma.

Rare or noble gases, such as helium, neon, argon and xenon. Plasma generated from other rare or noble gases, such as 


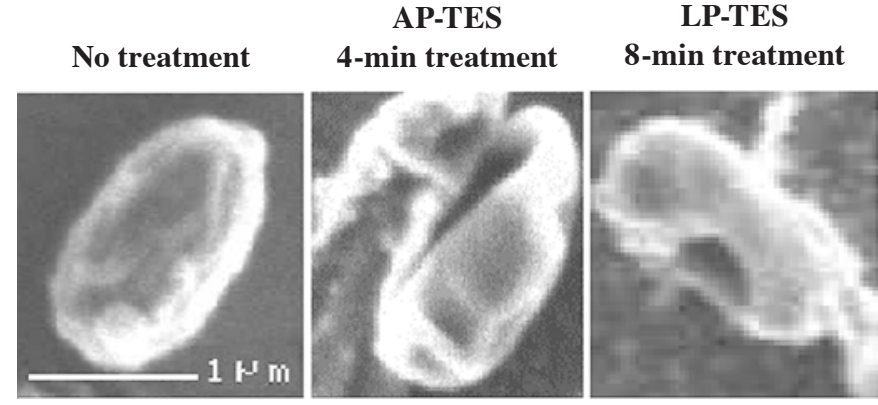

Figure 7. Scanning electron microscopic observation of the effects of pulsed $\mathrm{N}_{2}$ gas plasma exposure on a spore of G. stearothermophilus ATCC 7953 (1).
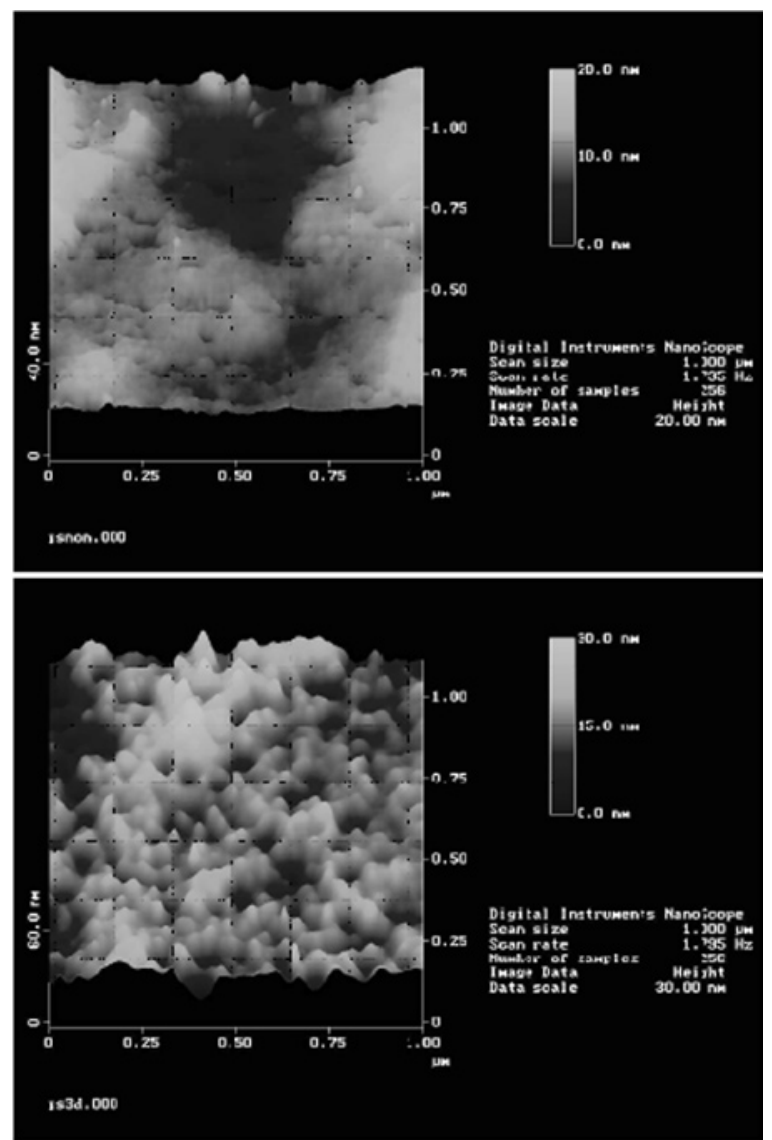

Figure 8 . Etching of a polystyrene surface by nitrogen gas plasma observed with atomic force microscopy (1). Upper panel, after 7 min of treatment; lower panel, control.

helium (He), neon (Ne), argon (Ar) and xenon (Xe), has also been studied (8-13). In a report (8) that presented a case for the use of Ar, the direct effects of Ar on exposed spores were similar to those observed with $\mathrm{N}_{2}$ gas plasma. Similar results were observed regarding $\mathrm{He}$ gas plasma, as shown in Figs. 9 and $10(9)$.

As rare gases are quite difficult to ionize, it is unlikely that the various ions, electrons, radicals or even UV contribute significantly as the active species in gas plasma sterilization exposures (9). In addition, a direct comparison to UV alone did not show any sporicidal effects (Fig. 10) (9).
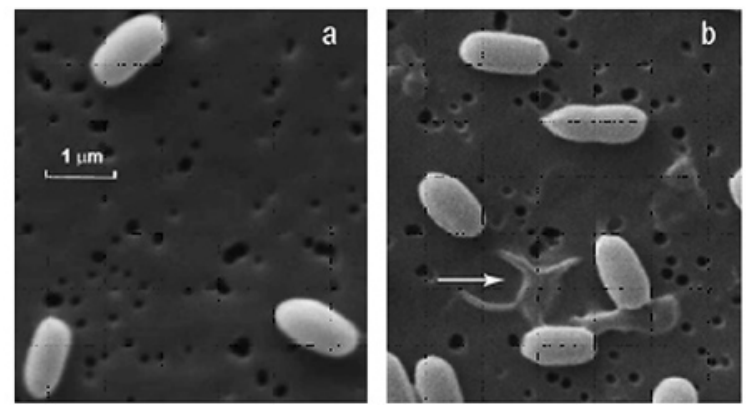

Figure 9. Scanning electron microscopic images of the B. atrophaeus ATCC 9372 spores (a) before He plasma treatment and (b) after He plasma treatment for $5 \mathrm{~min}$.

The effects of VUV should be considered (14-16), and indeed relevant research of the effects of exposure with VUV (at a 174-nm emission using an Ekishima UV apparatus from Iwasaki Electronic Co. Ltd., Japan) is underway (14).

As a whole, during $\mathrm{O}_{2}$ gas plasma exposure, it is not difficult to propose and define the active species present and responsible for the observed activity. However, the active species present in $\mathrm{N}_{2}$ and rare gas plasmas are more difficult to pinpoint and elucidate. It would appear that the $\mathrm{O}_{2}$ gas plasmas tested to date may have a significant disadvantage in terms of the deterioration of materials due to an etching phenomenon. By contrast, inert gases such as $\mathrm{N}_{2}$ or rare gases inactivate spores by a different mechanism, and are thus more desirable as they may achieve both the SAL efficacy requirements and material/functional compatibility required for validated sterilization processes. These may therefore prove to be useful alternatives to traditional sterilization methods such as humidified ethylene oxide gas, $\gamma$-ray, electron beam, steam sterilization and dry heat.

\section{Current uses of plasma for decontamination}

Decontamination is a general term that can be defined as any physical and/or chemical means to render a surface, instrument, tissue, liquid, air or indeed any item safe for handling, use or disposal (17). One typical example of cleaning by gas plasma is shown in Fig. 11 (1). 'Safe' in decontamination refers to reducing or completely removing/inactivating microorganisms that could lead to infection or other complications when inadvertently introduced into an individual. This includes numerous types of cleaning, disinfection and sterilization applications that are widely used in diverse medical, dental, agricultural, veterinarian, industrial and manufacturing practices. Various forms of plasma may have potential use in these applications. Currently, a variety of methods are used (17). These include physical methods, such as heat and radiation sources, as well as a range of chemicals, including hydrogen peroxide, glutaraldehyde and alcohols. Plasmas can be considered physico-chemical as they include a gas that in its own right may have some antimicrobial effects, but are also a physical source of energy.

To date, plasma decontamination applications have been limited to some industrial uses, and have not been widely adopted. There are currently no true commercially developed plasma-based decontamination processes, despite many inves- 


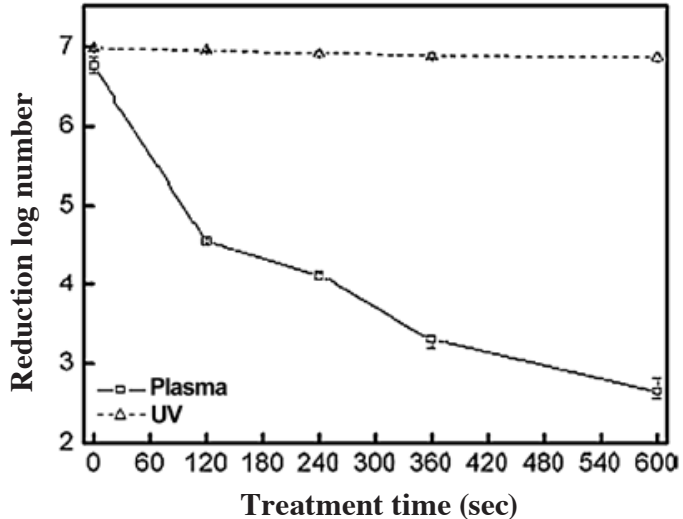

Figure 10. Spore population survivor curves of He gas plasma sterilization $(\square)$ in comparison to UV $(\triangle)(9)$. UV-C in this experiment had little to no effects on sterilization under the exposure conditions described.

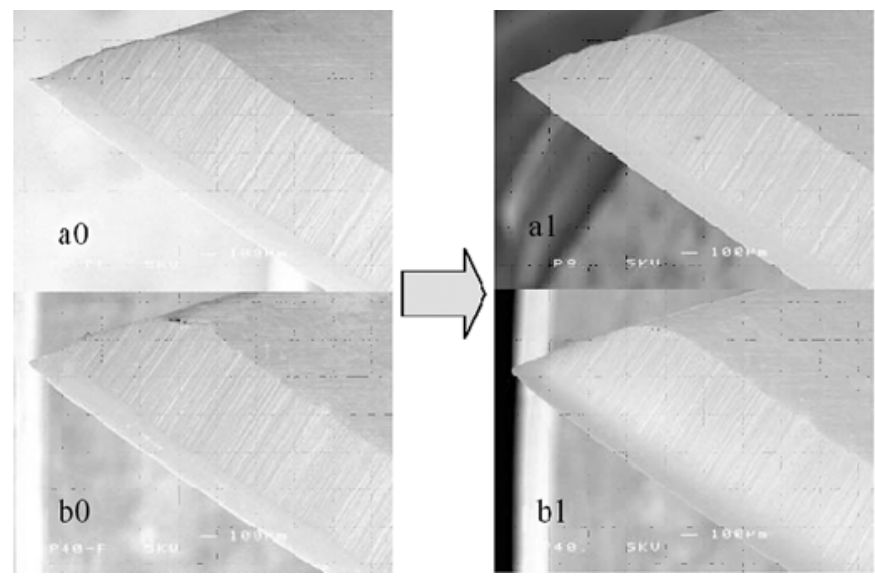

Figure 11. SEM observation of a stainless steel scalpel before and after $\mathrm{N}_{2}$ gas plasma exposure (1). Decontamination effects are observed in pulsed $\mathrm{N}_{2}$ gas plasma with no obvious damage to the surface of the sterilized material.

tigations and patents issued since the 1970s on various types of disinfection, sterilization and even cleaning applications. Notwithstanding the considerable research, the only processes that have been extensively used do not involve 'true' plasmas. In such processes, various types of gaseous oxidants, such as hydrogen peroxide, peracetic acid and ozone, are used to generate plasmas, but the plasma form is not used for antimicrobial purposes. In the case of the widely used hydrogen peroxide gas plasma sterilizers (STERRAD ${ }^{\circledR}$ systems), the cycle conditions clearly show that the plasma is only generated following exposure and removal of the gas (by vacuum) (2) and has little antimicrobial effect. However, in such processes, the plasma is important as it aids in the removal of peroxide (gas and liquid) residuals from the sterilizer load in order to make the contents safe for handling and use. Similarly, to date products that claim to use plasma to 'activate' water or other liquids and for decontamination simply use plasma as an alternative energy form to activate the various chemicals already present in (or added to) the water. Examples include the addition of $\mathrm{NaCl}$ to water, which activates the water to

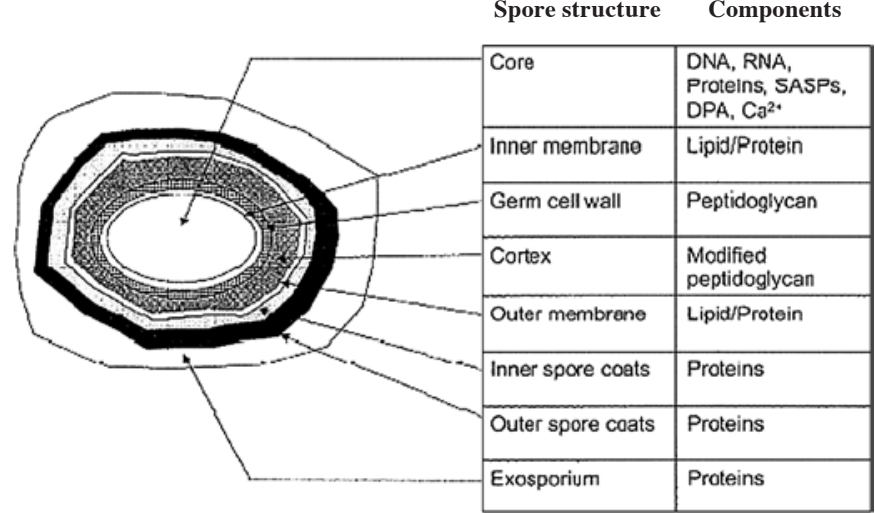

Figure 12. Representation of the interior and exterior structure of a bacterial spore (21). The inner membrane and DNA, SASP and DPA, Ca in the core are essential targets and indicators of effective sterilization.

make active free chlorine or hypochlorous acid. Indeed, in some investigations the mechanism of action has been clearly demonstrated to be chlorine rather than any true direct plasma effects (18).

A particularly active area of research over the last 5-10 years has been the elucidation and optimization of the various types and applications of true plasma-based antimicrobial processes. Potential applications have included, as an optimal cleaning process, combined cleaning-disinfection, terminal disinfection, and sterilization processes. In addition to industrial applications, these may have medical uses, such as in devices, drug-device combinations, tissues and air/water handling systems.

Once effective processes have been developed to apply plasma technology safely, effectively and practically, then commercial success can be achieved (19). Plasma technology has many advantages, such as broad spectrum antimicrobial activity (including effects against problematic biomolecules, such as prions and endotoxins), ease of generation, and environmental safety profiles in comparison to other thermal- and chemical-based technologies.

\section{Speculated mechanism of the action of gas plasma}

The mechanisms of action of gas plasma are clearly an area for further research. It has been suggested that they are related to various oxidation and reduction effects on the macromolecules that make up microbial structures (such as proteins, lipids and nucleic acids) (20). No studies published to date have directly demonstrated or clarified the definite mechanisms of gas plasma. Furthermore, as mentioned in this review, differences in the mechanisms of action have been reported between oxygen-based plasmas in comparison to other inert gases, such as $\mathrm{N}_{2}, \mathrm{He}$ and Ar.

Fig. 12 shows a representation of the external and internal structure of a bacterial endospore (20). Although these structures vary depending on the bacterial species and growth conditions, it is a useful representation for the purpose of this discussion. The inner spore membranes are quite rigid structures, where only molecules $<200$ daltons are capable of passing though. These membranes are important as they 


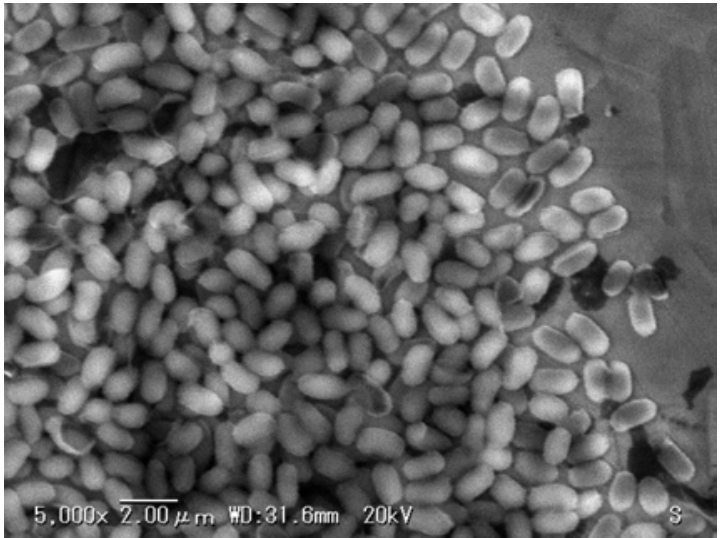

Figure 13. Scanning electron microscopy using A company biological indicator.

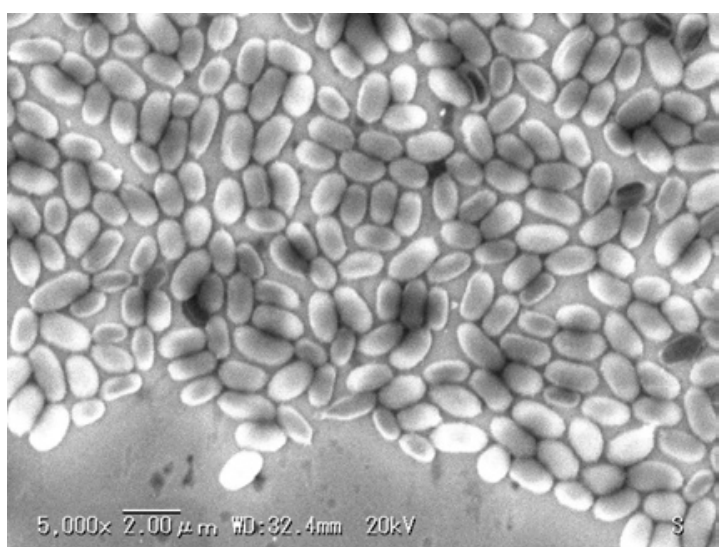

Figure 14. Scanning electron microscopy using B company biological indicator.

contain receptors for various germinants that promote germination and the outgrowth of the spore. Despite their importance, if the inner membrane is damaged, dipicolic acid (DPA) leaks out (as a clear indicator of both structural spore damage and initial germination), and germination is subsequently not promoted $(21,22)$. Access to these membranes is limited due to the various spore coats at the more external part of the spore structure, which require further damage/penetration in order for these inner membranes to be accessed. The inner core of the spore itself consists of various protection mechanisms, including the small acid soluble proteins that are tightly associated with and protect DNA from physical/ chemical attack. Thus, DNA damage is also an important factor to consider. Inert gases are inactive, but excited inert gas (metastable gas) molecules and radicals are expected to be capable of penetrating into the interior of the spore, without interfering with charged proteins and lipids surrounding the inner membrane and core. Excited molecules and radicals may therefore attack and pass though the inner membrane in order to attack the DNA at the core. In that sense, cations, anions, electrons, photons or UV-C (Figs. 1 and 2) are not speculated to be candidates for attacking the interior of the spore. Whether the inner membrane is damaged or not can be clarified by observation using a phase-contrast microscope

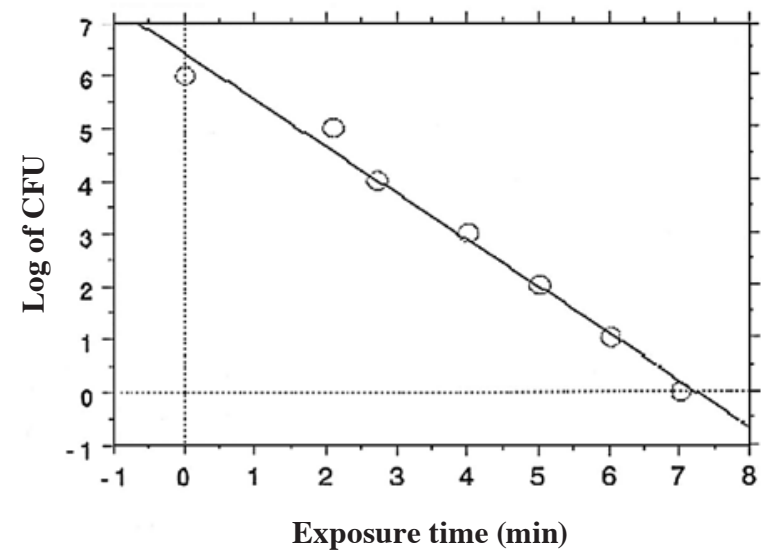

Figure 15. Plasma sterilization survivor curve using BI from B company presented in Fig. 14. The D value was approximately 1 min and no tailing phenomenon was reproducibly observed due to lack of clumping.

or scanning electron microscopy (23), and DPA release is determined in the culture medium of the surrounding area of the spore. Further experiments will be discussed in detail elsewhere (24).

By contrast, as proposed with other gas plasmas, the sporicidal effects are predominantly observed at the spore surface, leading to etching. These effects may initially cause spore surface damage (by oxidation-reduction reactions), but the affected proteins and other macromolecules then crosslink each other, leading to the loss of spore viability. Similar differences in efficacy have been reported for other sporicidal agents, even though they may be using the same biocide. For example, in the case of hydrogen peroxide, the mechanisms of action of hydrogen peroxide in a true gas form appear to be different from those of liquid or condensed peroxide $(18,19)$. The gas form appears to primarily target the peptide bonds of proteins, leading to protein degradation; by contrast, the liquid form preferably oxidizes the amino acid side chains of the same proteins, which appears to result in cross-linking and protein clumping. These effects are further complicated in the presence of other (inert) chemistries (as is common when using hydrogen peroxide), and there has also been a report of other oxidizing agents.

Overall, the true mechanisms of action of most biocides against bacterial spores are not very well described in the literature, while an area for future research should include the study of these mechanisms with various plasmas. Experiments that investigate the direct effects of plasma on the macromolecules that make up microbial structures may be an initial and significant way to enhance the understanding of these effects under controlled conditions.

\section{Sporicidal testing and the importance of spore clumping}

Almost all studies investigating the kinetics of sporicidal activity with plasmas published to date present the same tailing phenomenon in survivor curves $(9,25)$. The structure of such survivor curves is an important consideration in differentiating a disinfection process from a sterilization process, and particularly in allowing the extrapolation of sterility assurance levels. 
Microorganism

Examples

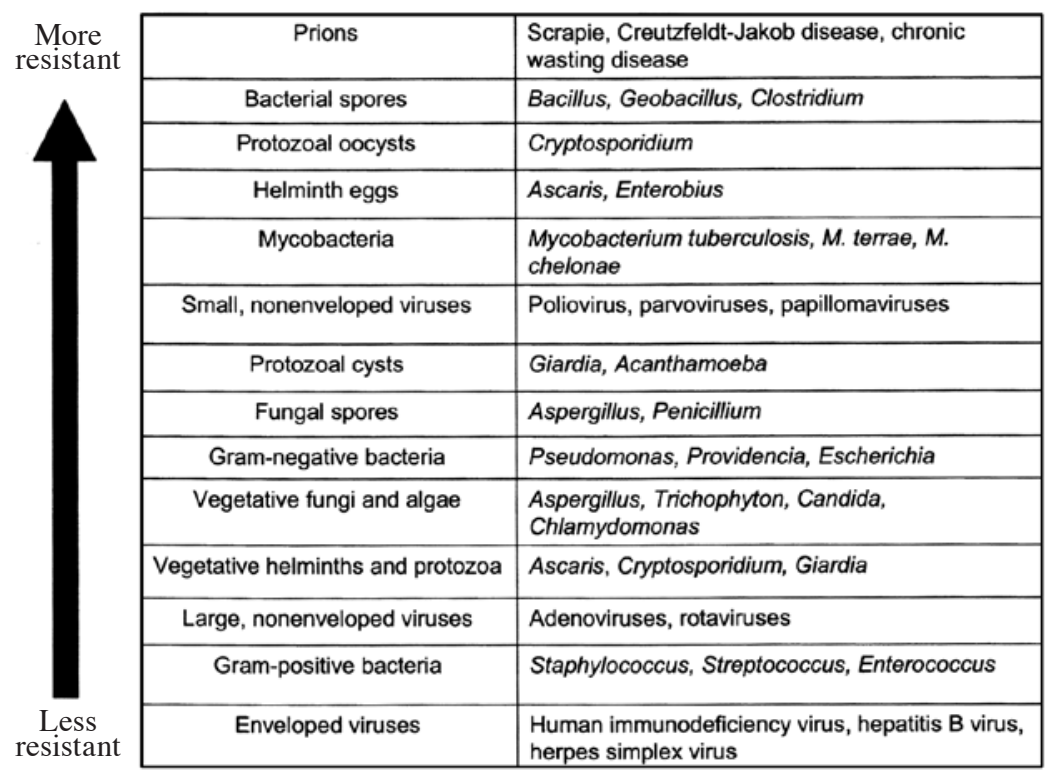

Figure 16. A guide to microbial resistance to biocides and biocidal processes (20). Various types of microorganisms are listed, being less resistant (more sensitive) and more resistant as you move up the list. Vegetative (actively growing) forms are generally more sensitive than the more resistant dormant forms (such as protozoal oocysts and bacterial spores). The unusual infectious agents, known as 'prions', are considered to demonstrate even higher resistance, although recent evidence would suggest that this may not always be the case. It is important to note that this list can only be used as a guide, as the exact resistance profile will vary depending on the antimicrobial active and even the exact product/process under investigation.

Tailing in plasma survivor curves is shown by an initial linear relationship between log reduction of spore populations over time, followed by a non-linear relationship in the quantal region of the kill curve (Fig. 10). This would prevent the extrapolation of an SAL of $10^{-6}$ at an initial population of $10^{6} \mathrm{CFU}$ required for an overkill sterilization process. This can be interpreted as a limitation in the antimicrobial process under testing and/or an artifact of the test method. The most common cause of artifacts is clumping of the spore preparations used for testing (7).

The most recognized resistant organism for steam sterilization, hydrogen peroxide gas, peroxide gas plasma and potentially other plasmas (to date) are the endospores of Geobacillus stearothermophilus ATCC 7953 or 12980. During sterilization investigations, spore suspensions of $G$. stearothermophilus at a population of $10^{6} \mathrm{CFU}$ are inoculated onto appropriate carrier materials, such as stainless steel or polystyrene. These are commercially available as BIs (Figs. 13 and 14). In plasma experiments, BIs from one manufacturer (A) demonstrated a reproducible tailing phenomenon, which was assumed to be due to clumping (due to spores being present in multilayers; Fig. 13). By contrast, the survival curve observed by another manufacturer (B) (Fig. 14) using the same plasma process presented a reproducible linear relationship (Fig. 15), allowing for the extrapolation of a SAL. The D-value (the average time to obtain a log reduction of the test microorganism) calculated from Fig. 15 was approximately $1 \mathrm{~min}$. Therefore, a minimum sterilization time of $12 \mathrm{~min}$ for overkill method under these exposure conditions would provide a SAL of $10^{-6}$, as required by the international standards for sterilization processes (ISO 14937) (3). The coefficient correlation between log reduction of the test microorganism and exposure time was 0.992 (Fig. 15). This relationship is quite satisfactory for the extrapolation of a SAL.
The overkill method of demonstrating a sterilization process is the most widely used method, but not the only one. Sterilization is also demonstrated by an understanding of the natural bioburden (numbers and types of viable microorganisms) present on/in the product to be sterilized; this is indeed the real target for sterilization. When the bioburden is low, the sterilization challenge is not great, but when it is high and associated with significant clumping (such as observed with viruses and various types of bacteria, including the presence of biofilm) (26), this provides a significant challenge to the sterilization process. However, in many cases these vegetative bacterial cells (27) and other microorganisms are generally much less resistant to gas plasmas (20) (Fig. 16). Therefore, the gas plasma penetration ability, as previously discussed, should be sufficient to penetrate and sterilize any such bioburden present and to simultaneously attain an appropriate SAL of $10^{-6}$ together with material and functional compatibility. This needs to be demonstrated for any potential plasma sterilization process.

\section{Perspectives for the future}

It seems likely that gas plasma disinfection and/or sterilization methods can be developed to provide the required antimicrobial reduction (as defined by an appropriate SAL, e.g., $10^{-6}$ for sterilization) and material/functional compatibility. Plasma may have important applications in medical and industrial settings. In this sense, perspectives on plasma sterilization methods require further investigation. To clarify the mechanisms and optimize the development of gas plasma sterilization, close collaboration between microbiologists and physical chemists will provide the best opportunity for the future success of this technology. 


\section{References}

1. Shintani H, Shimizu N, Imanishi Y, Sekiya T, Tamazawa K, Taniguchi A and Kido N: Inactivation of microorganisms and endotoxins by low temperature nitrogen gas plasma exposure. Biocontrol Sci 12: 131-143, 2007.

2. Krebs MC, Becasse P, Verjat D, et al: Gas plasma sterilization: relative efficiency of the hydrogen peroxide phase as compared to that of the plasma phase. Int J Pharm 160: 75-81, 1988.

3. ISO 14937: 2009. Sterilization of health care products. General requirements for characterization of a sterilizing agent and the development, validation and routine control of a sterilization process for medical devices.

4. Sato N: Basic approach to plasma production and control. In: Advanced Plasma Technology. D'Agostino R, Favia P, Kawai Y, Ikegami H, Sato N and Arefi-Khonsari F (eds). Wiley-VCH, Verlag GmbH \& Co. Weinheim, Germany, pp1-16, 2008.

5. Kylian O, Sasaki $\mathrm{T}$ and Rossi F: Plasma sterilization of Geobacillus stearothermophilus by $\mathrm{O}_{2}: \mathrm{N}_{2} \quad \mathrm{RF}$ inductively coupled plasma. Eur Phys J Appl Phys 34: 139-142, 2006.

6. Jacobs PT and Lin S-M: Sterilization processes utilizing low-temperature plasmas. In: Disinfection, Sterilization and Preservation. Block SS (ed). Lippincott Williams \& Wilkins, New York, pp747-765, 2001.

7. Rossi F, Kylian O and Hasiwa M: Mechanisms of sterilization and decontamination of surfaces by low-pressure plasma. In: Advanced Plasma Technology. D'Agostino R, Favia P, Kawai Y, Ikegami H, Sato N and Arefi-Khonsari F (eds). Wiley-VCH Verlag GmbH \& Co., Weinheim, Germany, pp319-340, 2008

8. Rossi F, Kylian O and Hasiwa M: Decontamination of surfaces by low pressure plasma discharges. Plasma Process Polym 3: 431-442, 2006.

9. Deng X, Shi J and Kong MG: Physical mechanisms of inactivation of Bacillus subtilis spores using cold atmospheric plasmas. IEEE Trans Plasma Sco 34: 1310-1316, 2006.

10. Kim S-M and Kim J-I: Decomposition of biological macromolecules by plasma generated with helium and oxygen. J Microbiol 44: 466-471, 2006.

11. Lee K, Paek K-H, Ju W-T and Lee Y: Sterilization of bacteria, yeast, and bacterial endospores by atmospheric-pressure cold plasma using helium and oxygen. J Microbiol 44: 269-275, 2006.

12. Yu QS, Huang C, Hsieh F-H, Huff H and Duan Y: Bacterial inactivation using a low-temperature atmospheric plasma brush sustained with argon gas. J Biomed Mater Res Part B: Appl Biomater 80B: 211-219, 2007.

13. Purevdorj D, Igura N, Ariyada O and Hayakawa I: Effect of feed gas composition of gas charge plasmas on Bacillus pumillus spore mortality. Lett Appl Microbiol 37: 31-34, 2003.
14. Kinoshita S: Examination of UV and VUV effect on sterilization. Bohkin Bobai (In press).

15. Halfmann H, Bibinov N, Wunderlich $\mathrm{J}$ and Awakowicz $\mathrm{P}$. Correlation between VUV radiation and sterilization efficiency in a double inductively coupled plasma. Presented at the 28th ICPIG Congress. Prague, 2007

16. Lerouge S, Fozza AC, Wertheimer MR, Marchand $\mathrm{R}$ and Yahia L: Sterilization by low-pressure plasma: the role of vacuum-ultraviolet radiation. Plasma Polymers 5: 31-46, 2000.

17. Güçeri S and Fridman A: Plasma Assisted Decontamination of Biological and Chemical Agents. Springer, The Netherlands, 2008.

18. McDonnell G: Peroxygens and other forms of oxygen: their use for effective cleaning, disinfection and sterilization. In: New Biocides Development: the Combined Approach of Chemistry and Microbiology. Zhu PC (ed). Oxford University Press, New York, pp292-308, 2006.

19. Finnegan M, Linley E, Denyer SP, McDonnell G, Simons C and Maillard J-Y: The mode of action of hydrogen peroxide and other oxidizing agents: differences in liquid and gas form. J Pharm Sci (In press).

20. McDonnell GE: Antisepsis, Disinfection, and Sterilization. ASM Press, Washington DC, pp33,282, 2007.

21. Cortezzo DE, Koziol-Dube K, Setlow B and Setlow P: Treatment with oxidizing agents damages the inner membrane of spores of Bacillus subtilis and sensitizes spores to subsequent stress. J Appl Microbiol 97: 838-852, 2004.

22. Setlow P: Spores of bacillus subtilis: their resistance to and killing by radiation, heat and chemicals. J Appl Microbiol 101: 514-525, 2006.

23. Imae Y, Strominger MB and Strominger J: Electron microscope studies of conditional spore cortexless mutants of Bacillus sphaericus. J Bacteriol 127: 1568-1570, 1976.

24. Shintani $\mathrm{H}$ and McDonnell G: Inactivation of microorganisms (spore type and vegetative cells) and the mechanism by gas plasma. In: Sterilization and Disinfection by Plasma: Sterilization Mechanism, Biological and Medical Applications. Sakudo A and Shintani H (eds). NOVA Science Publishers, New York (In press).

25. Moisan M, Barbeau J, Moreau S, Pelletier J, Tabrizian M and Yahia LH: Low-temperature sterilization using gas plasmas: a review of the experiments and an analysis of the inactivation mechanism. Int J Pharm 226: 1-21, 2001.

26. Joaquin JC, Kwan C, Abramzon N, Vandervoort $\mathrm{K}$ and Breiles-Marino G: Is gas-discharge plasma a new solution to the old problem of biofilm inactivation? Microbiology 155: 724-732, 2009.

27. Shintani H, Taniai E, Miki A, Kurosu S and Hayashi F: Comparison of the collecting efficiency of microbiological air samplers. J Hosp Infect 56: 42-48, 2004. 